\title{
DIGITAL HEARING AID SIGNAL PROCESSING SYSTEM USING ANDROID PHONE
}

\author{
YEH-HUANN GOH ${ }^{*}$, YOON-KET LEE ${ }^{1}$, MUM-WAI YIP ${ }^{1}$, KOK-SENG E. U. ${ }^{1}$, YANN LING GOH ${ }^{2}$, KIN-YUN LUM ${ }^{1}$
}

${ }^{1}$ Department of Mechanical Engineering, Faculty of Engineering and Technology, Tunku Abdul Rahman University College, Jalan Genting Kelang, Setapak, 53300 Kuala Lumpur, Malaysia. ${ }^{2}$ Department of Mathematical and Actuarial Sciences, Lee Kong Chian Faculty of Engineering and Science, Universiti Tunku Abdul Rahman, Sungai Long Campus, 43000, Kajang, Selangor, Malaysia. Email: gohyh@tarc.edu.my

Received: 03 November 2018, Revised and Accepted: 30 November 2018

ABSTRACT

Objective: The objective of this research is to propose an Android-based digital hearing aid signal processing algorithm with following key features: (1) Regenerated audio match the patient-specific pattern of hearing loss, (2) noise reduction, and (3) provide flexibility to the users.

Methods: The proposed signal processing algorithm is designed based on the specific hearing loss of the hearing disorder patient using inverse Fourier transform; besides, noise reduction feature is included in the digital algorithm design as well. Proposed digital algorithm has been implemented into an Android-based smartphone and its performance has been tested under real-time condition.

Results: Simulation results show that the frequency response of the proposed digital hearing aid signal processing algorithm is in agreement with the initial theoretical design that was carried out based on the hearing impaired patient's audiogram. The proposed algorithm has been implemented in the Android-based smartphone and tested in real time. Results show that most of the patients are satisfied with the regenerated audio quality. According to patient's comments, the regenerated audio is clear and the users are allowed to control the volume level. Besides, no obvious hearing latency can be detected.

Conclusion: Audio signals generated by the proposed digital signal processing algorithm show similar audio signal frequency response in both theoretical design and MATLAB simulation results. The only difference between the design and simulation results is the amplification levels. The proposed algorithm provides flexibility to the users by allowing them to choose the desired amplification level. In real-time testing, the proposed Android-based digital hearing aid is able to reduce noise level from the surrounding and the output processed speech match the patient-specific hearing loss.

Keywords: Digital hearing aid, Digital algorithm, Signal processing, Android phone.

(C) 2019 The Authors. Published by Innovare Academic Sciences Pvt Ltd. This is an open access article under the CC BY license (http://creativecommons. org/licenses/by/4. 0/) DOI: http://dx.doi.org/10.22159/ijap.2019.v11s5.T3014

\section{INTRODUCTION}

Hearing aids act as the social integration of people that suffer from hearing limitations or neurosensorial losses [1]. The estimated number of individuals with some form of hearing impairment ( $20 \mathrm{~dB}$ or more) rose from 807.2 million in 1990 to 1.23 billion in 2013 [2]. Wrood et al. shows that co-exposures of cadmium and lead in environment will cause a significant elevation of cadmium and lead level in the human blood that could results in hearing impairment [3]. Hearing aid is a very complex system that consists of several processing units that perform tasks such as adaptive directionality, noise reduction, dynamic compression, feedback cancellation, and others that help to improve the intelligibility and also at the same time provide better acoustic comfort for the user. Due to their small size and low-power consumption, the availability of the computational resources for each of the subsystem has been restricted [1].

The advance in digital signal processing (DSP) and microelectronics has led to the development of the superior digital hearing aid for the purpose of improving the severe effects of hearing. Hearing aids that utilize the concepts of DSP are able to provide desirable features that could not be realized with any conventional ear-level analog hearing aids in the aspects of the physical size and power consumption limitations [4]. Individually tuned hearing aids can satisfy most of the requirements of hearing impaired persons. A multiband loudness correction algorithm for a digital hearing aid has been proposed by optimizing the parameters of the adaptive beamformer noise reduction algorithm in Dillier et al. [5]. The progress in the field programmable gate arrays (FPGA) technology has allowed the increasing in sophisticated features to be built to have the results of better sound reproduction, keeping size smaller, and lowering down the power consumption of the devices. Digital hearing aids were designed by combining the FPGAs with the embedded microprocessors plus the related peripherals to form a complete system on a programmable chip [6]. Various speech coding strategies have been developed for cochlear implants in real-time configuration based on a DSP laboratory speech processor in Kiefer et al., [7] Nogueira et al., [8] Skinner et al. [9].

Recently, different techniques have been applied in the hearing aid design such as wavelet packet transform for speech compensation of the hearing loss in Ghamry [6]. An improved partitioned block Hartley domain adaptive filter was applied for effective feedback cancellation in hearing aids to reduce the computational burden and simultaneously retaining its performance in Panda and Puhan [10]. Spatial filtering method computes the optimized filter coefficients with the purpose of maximizing the array gain and at the same moment minimizing the distortions in Ayllón et al. [11]. Efficient and simple designs of non-uniform filter banks for digital hearing aid applications were implemented in Kalathil and Elias [12].

Hearing aid devices are normally portable and battery operated. Several low-power consuming hearing aid devices have been proposed. Lowpower consumption, high-performance analog front-end circuit for digital hearing aid which adopts digital-feedback-gain-control for accurate amplification and the multi-bit Sigma-Delta modulator technique to improve dynamic range has been proposed in Chen et al. [13]. A low-power real-time binaural wearable digital hearing aid platform was developed based on the TMS320C5000 ${ }^{\mathrm{TM}}$ fixed-point DSP in Stetzler et al. [14]. 
The advancement of Android smartphone allows it to be used in various fields such as signal processing field [15]. Available speech processing functions in Android application include generating spectrogram, spectrum analyzer, text to speech conversion, and speech to text conversion. An Android smartphone is essentially a small screen computer, it allows user to carry out a wide range of tasks when on the go. The proposed hearing aid DSP algorithm will be implemented in the smartphone and the smartphone is being used as a hearing aid device.

The organization of the paper is as follows: Section 2 begins with a brief review of the sampling rate of the speech signals in different application areas. This followed by the design of a digital hearing aid signal processing algorithm based on a general audiogram of the presbyacusis hearing impaired disease. Besides, other features such as (1) noise reduction and (2) adjustable levels of amplification are included in the study. Section 3 shows the comparison of the initial theoretical design and the simulation results. In Section 4, the proposed algorithm has been implemented into an Android-based smartphone; performance of the Android-based digital hearing aid has been tested under real-time condition. Section 5 presents the conclusion of this study.

\section{Digital hearing aid signal processing design}

The audio signal data rate needed for an audio signal will affect the quality of the reproduced sound. A low-end telephone quality speech requires capturing the frequencies between $100 \mathrm{~Hz}$ and $3.2 \mathrm{kHz}$ at a sampling rate of $8 \mathrm{k}$ samples/s. Full audio frequency range of human hearing is needed for high fidelity music, which is from $20 \mathrm{~Hz}$ to $20 \mathrm{kHz}$. For both the left and right channels, $44.1 \mathrm{kHz}$ sampling rate is used and this makes the complete high fidelity signal sampling rate to be $88.2 \mathrm{k}$ samples/s [16].

Although audio signals with high sampling rate improves the speech quality, however, for high sampling rate, longer processing time is needed for a hearing aid to reproduce the audio signals. Since the quality of a telephone speech signals is widely acceptable by the public, the sampling rate of the audio signals used in the DSP algorithm proposed in this paper is chosen to be $8 \mathrm{kHz}$.

\section{Features of the design digital hearing aid algorithm}

The voiced speech of a typical adult male will have a fundamental frequency ranging from 85 to $155 \mathrm{~Hz}$ and for a typical adult female is from 165 to $255 \mathrm{~Hz}$ [17]. Besides, the upper band frequency of a telephone speech is $3.2 \mathrm{kHz}$; a bandpass filter with passband ranging from $85 \mathrm{~Hz}$ to $3.2 \mathrm{kHz}$ is included in digital hearing aid signal processing algorithm design for noise reduction purpose. Received audio signals with frequencies lower than $85 \mathrm{~Hz}$ or higher than $3.2 \mathrm{kHz}$ are considered as noise and get filtered out by the proposed digital hearing aid.

Proposed digital hearing aid signal processing algorithm processes the received audio signals and reproduces the audio signals in the form that match the patient-specific pattern of hearing loss. The specific pattern of the patient hearing loss is predetermined from the audiometric examination test. In this paper, a DSP algorithm has been designed for a presbyacusis patient by referring to a general presbyacusis hearing disorder audiogram.

\section{Presbyacusis}

Presbyacusis is a type of hearing disorder which is associated with aging process. It usually affects high frequencies range than the low frequencies range. Fig. 1 shows a general audiogram of a presbyacusis hearing disorder patient [18].

Circles in Fig. 1 represent the patient's right ear hearing sensitivity and the crosses represent the patient's left ear hearing sensitivity. By referring to Fig. 1, less amplification is needed for frequency smaller than $1 \mathrm{kHz}$. However, when the audio frequency gets higher than $1 \mathrm{kHz}$, for the patient to hear the audio, higher amplification in audio signals is needed. The proposed signal processing algorithm is designed based on the level of hearing loss in different frequencies of sound observed in the audiogram.

\section{Design of digital hearing aid processing system}

A finite impulse response (FIR) filter is designed based on the frequency response specification. The equivalent sampled impulse response, which determines the coefficients of an FIR filter, is found by inverse (discrete) Fourier transformation.

Fig. 2 shows the frequency response specification design of the proposed digital hearing aid signal processing algorithm. This design includes the following features: (1) Bandpass filter with passband ranging from 85 $\mathrm{Hz}$ to $3.2 \mathrm{kHz}$ and (2) output frequency gain that matches the patient's specific pattern of hearing loss as shown in Fig. 1. Different levels of amplification at different frequencies can be observed in Fig. 2. The amplification is lower at low-frequency region and higher at the highfrequency region. Besides, the design frequency response specification provides noise reduction function; all the voice signals with frequency level lower than $85 \mathrm{~Hz}$ or higher than $3.2 \mathrm{kHz}$ are considered as noise and will be filtered out. A smoother frequency response can be designed using more frequent and smaller step increment in the frequencyresponse design.

XBy substituting the design frequency response specification into Equation (1) and perform the inverse Fourier transform,

$h(t)=\int_{-\alpha}^{\alpha} H(f) e^{j 2 \pi f t} d f$

The transfer function of the design digital hearing aid signal processing algorithm is obtained as,

$h(t)=\frac{1}{\pi t}\left[\begin{array}{l}1.6 \sin 6400 \pi t-0.1 \sin 6000 \pi t \\ -0.1 \sin 5000 \pi t-0.1 \sin 4000 \pi t \\ -0.1 \sin 3000 \pi t-0.1 \sin 2000 \pi t-1.1 \sin 170 \pi t\end{array}\right]$

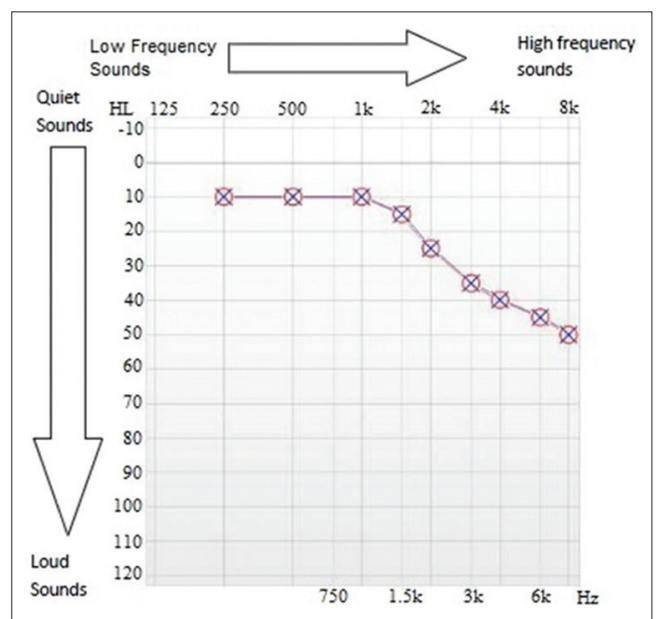

Fig 1: General audiogram of the presbyacusis hearing impaired disease

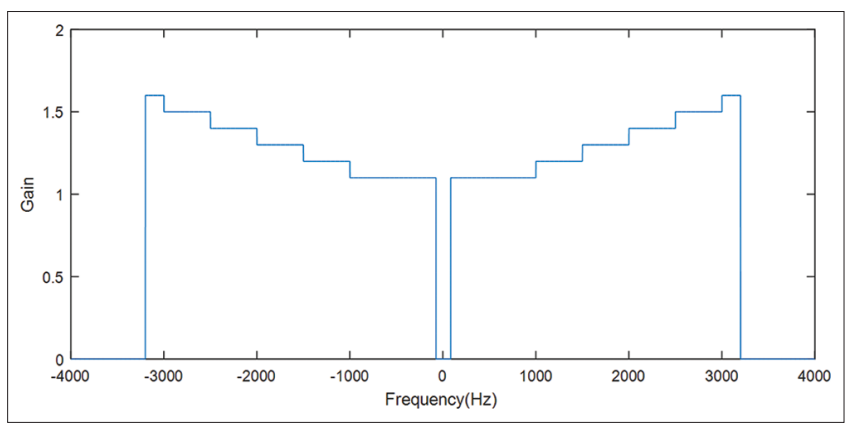

Fig. 2: Frequency response-design of the proposed digital hearing aid signal processing algorithm 
When sampling rate is equal to $8 \mathrm{kHz}$, the digital sequence of the design filter is as follows:

$$
h(t)=\frac{K}{\pi n}\left[\begin{array}{l}
1.6 \sin 0.8 \pi n-0.1 \sin 0.75 \pi n \\
-0.1 \sin 0.625 \pi n-0.1 \sin 0.5 \pi n \\
-0.1 \sin 0.375 \pi n-0.1 \sin 0.25 \pi n-1.1 \sin 0.02125 \pi n
\end{array}\right]
$$

When $\mathrm{n}=0$, using squeeze theorem, $\mathrm{h}(0)$ is found to be $0.796 \mathrm{~K}$, where $\mathrm{K}$ is a constant number. Constant number $\mathrm{K}$ is included in Equation (3) since the theoretical calculation results may be different from the realtime signal processing results. The signal processing function at current state $h(0)$ is fixed to 1 ; the value of $K$ is found to be 1.256 . Fig. 3 shows the plot of the function h(n).

To obtain the real-time performance of the digital filter similar to the theoretical design in Fig. 2, infinite number of coefficients is needed. However, in Fig.3, the magnitude of $\mathrm{h}(\mathrm{n})$ becomes smaller when the value of $|\mathrm{n}|$ becomes larger. This makes the effects of the speech signals at the far future and past time states not significant. Besides, longer processing time is needed to filter the speech signals when the number of coefficients used is larger. One hundred and sixty-one $h(n)$ coefficients ranging from $-80 \leq n \leq 80$ are used in the design algorithm. Equation (4) shows the convolution process of speech signal model s(n) and the signal processing function $\mathrm{h}(\mathrm{n})$.

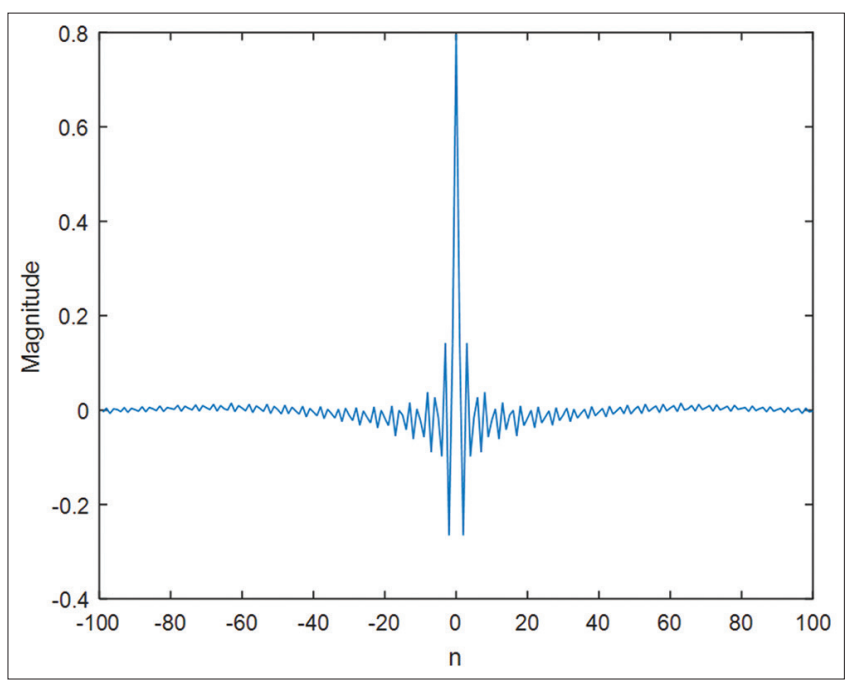

Fig. 3: Digital hearing aid signal processing function $h(n)$

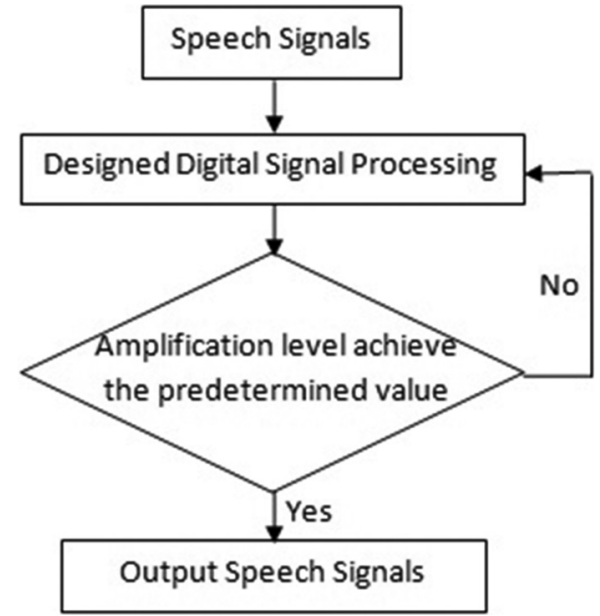

Fig. 4: Hearing aid speech signal processing block diagram

$$
[s \times h](n)=\int_{-80}^{80} s(\tau) h(n-\tau) d \tau
$$

Compared to original speech signals, output of Equation (4) is the reproduced audio signals with lower noise level and its amplification levels match the audiogram pattern better. Fig. 4 shows the block diagram of the proposed DSP hearing aid algorithm flow. Initially, users are required to choose the level of amplification. A total of four amplification levels can be selected from the device. After selecting the level of amplification, the proposed hearing aid captures the audio signals from the surrounding and processes the audio signals using Equation (4). By increasing the number of iteration, higher level of amplification can be achieved. When the amplification level of the audio signals reaches the predetermined level, hearing aid reproduces the processed audio signals and plays the audio.

\section{RESULTS AND DISCUSSION}

The proposed digital hearing aid processing algorithm was tested using MATLAB simulation results. Comparison was made between the initial theoretical frequency response specification design and the simulation results. Fig. 5 shows the frequency response plots of the initial design and the simulation results in MATLAB. Simulation results show a similar frequency response with the initial theoretical design. However, simulation results are different from the initial design in the aspect of amplification levels. The amplification levels for frequency higher than $1 \mathrm{kHz}$ are higher than the original design.

Fig. 6 shows different amplification levels in decibel when speech signal is processed by different iteration numbers. When iteration number is increasing, attenuation levels for audio signals with frequency components lower than $85 \mathrm{~Hz}$ and higher than $3.2 \mathrm{kHz}$ become larger. Amplification levels at different frequencies get higher follow the increasing in iteration number. In theoretical design, the amplification levels start from $10 \mathrm{~dB}$ at low-frequency region and ramping up to 40 $\mathrm{dB}$ at $3.2 \mathrm{kHz}$. From the obtained simulation results, at the $4^{\text {th }}$ iteration,

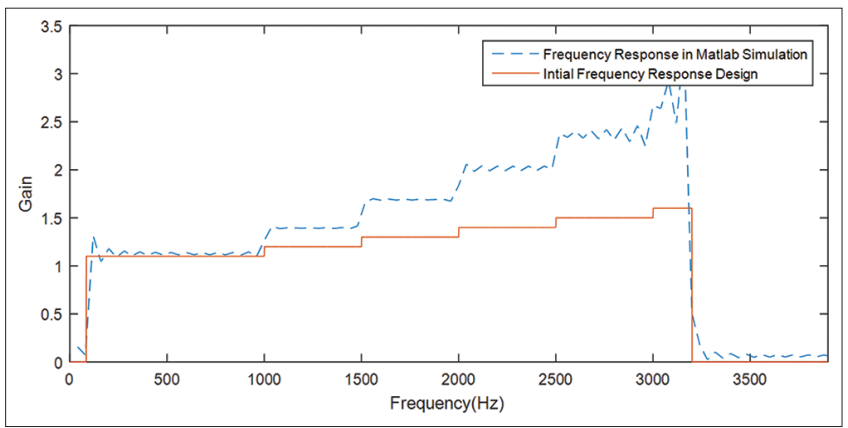

Fig. 5: Frequency response plots for initial theoretical design and final simulation results at the $1^{\text {st }}$ iteration

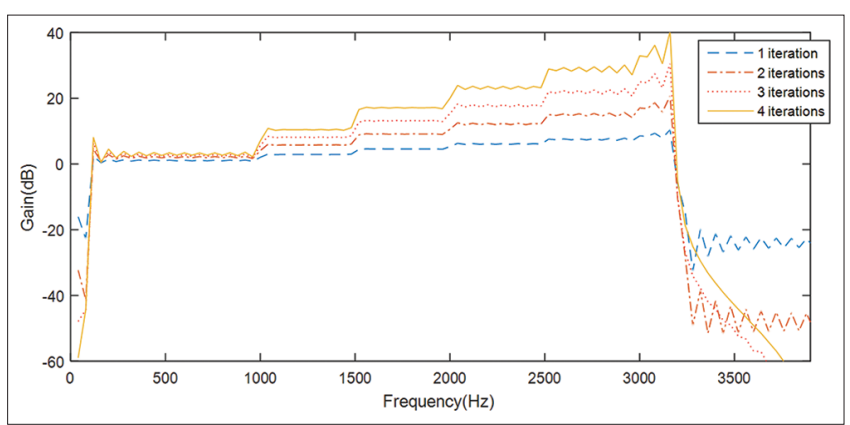

Fig. 6: Frequency response of the proposed digital hearing aid signal processing algorithm at different iteration numbers 


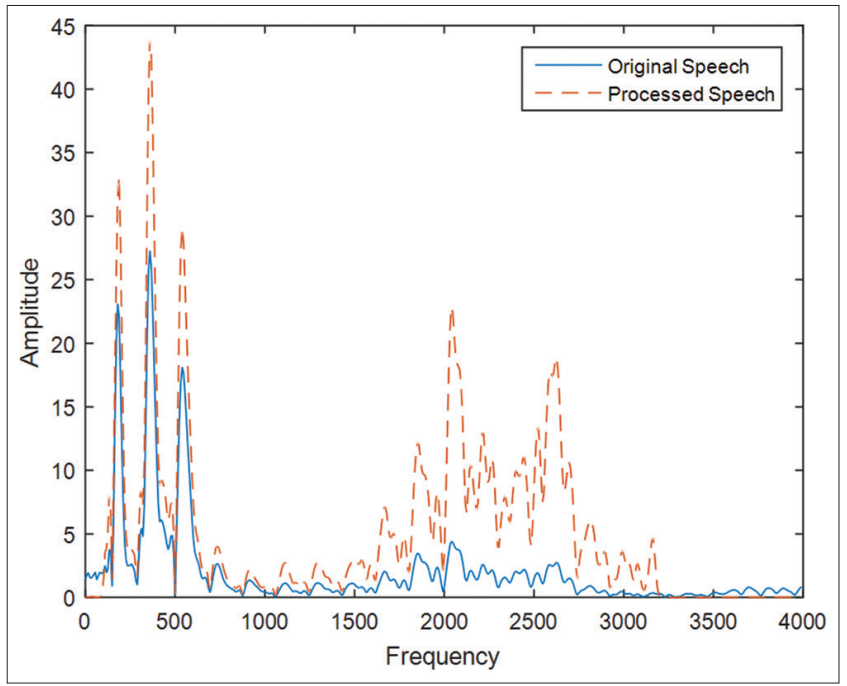

Fig. 7: Discrete Fourier transform of original speech signals and processed speech signals "six" spoken by a female speaker

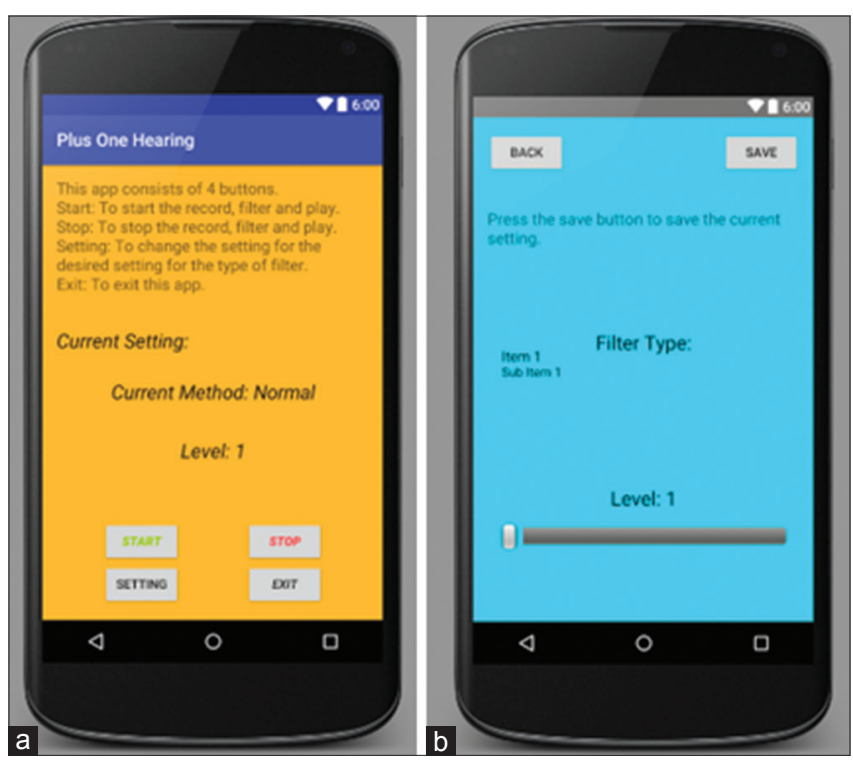

Fig. 8: (a) Main activity interface created in Android Studio,

(b) setting activity interface created in Android Studio

for low-frequency region ( $85 \mathrm{~Hz}-1 \mathrm{kHz}$ ), amplification level is $4 \mathrm{~dB}$. The amplification levels ramp up to $40 \mathrm{~dB}$ at $3.2 \mathrm{kHz}$. The frequency response of the proposed digital hearing aid algorithm matches the presbyacusis hearing disorder patient audiogram pattern as shown in Fig. 1. A smoother frequency response can be obtained by designing a smoother increment in the preliminary theoretical frequency response in Fig. 2.

Fig. 7 shows two discrete Fourier transform plots of a speech signal six, before and after being processed by the proposed digital hearing aid signal processing algorithm using four iterations amplification. It was spoken by a female speaker, and the word six consists of both high-frequency unvoiced components and low-frequency voiced components. Fig. 7 clearly indicates that speech signals with frequency lower than $85 \mathrm{~Hz}$ and higher than $3.2 \mathrm{kHz}$ are being filtered out by the digital filter. Besides at low-frequency region $(\mathrm{f}<1$ $\mathrm{kHz}$ ), amplification level is smaller than 2. At high-frequency region, amplification level becomes larger and larger follows the increment in frequencies. This amplification trend follows the initial theoretical design.
Table 1: Feedbacks from senior citizens

\begin{tabular}{lll}
\hline Clarity & Loudness & Latency \\
\hline 80\% better & 80\% better & $100 \%$ undetectable \\
$20 \%$ no different & $20 \%$ no different & $0 \%$ detected \\
$0 \%$ poorer & $0 \%$ poorer & \\
\hline
\end{tabular}

Real-time Android-based digital hearing aid

Android Studio is used to create an app for both the DSP algorithm and interface. In Android Studio, there are two main types of coding platform file type.,XML file type coding and.JAVA file type coding.

In Android Studio, the interface of each page is called activity. The created app consists of two activities which are main activity and setting activity. Interface is created using.xml file type coding. The main activity interface consists of a guide for user to understand on how to use the app, text box to display the current user setting, and four buttons. The four buttons are Start button, Stop button, Setting button, and Exit button. Function of Start button is to start capturing the audio signals using a microphone and then processes the audio signals digitally and plays the audio signals back in real time. Function of Stop button is to stop the hearing aid function but stay in the digital hearing aid app. Function of Setting button is to open the setting activity. Function of Exit button is to exit the digital hearing aid app. Fig. 8 shows the activity pages of the proposed hearing aid.

Setting activity provides the flexibility to the users. Users are allowed to change the setting according to their needs. In setting activity, there are two buttons, a seekbar, a spinner and text boxes for guidance and indication. The function of Back button is to return to the main activity page. Function of Save button is to save the preference of the users. Spinner is a drop-down list that shows the list of choices related to type of hearing disorder. Multiple digital hearing aid signal processing algorithms designed based on different hearing disorder patterns can be saved in the hearing aid app. The function of seekbar is to set the amplification level. The available levels are from 1 to 4 .

After coding, Android Studio builds the code into an app and the app is installed into the Android smartphone. The performance of the Android smartphone-based hearing aid is tested under real-time condition. The hearing latency of the device is undetectable by the users. Noise level of the surrounding has been reduced due to the bandpass filter from $85 \mathrm{~Hz}$ to $3.2 \mathrm{kHz}$ available in the digital processing design. The amplification level for the unvoiced speech signals which consists of high-frequency components is significantly higher than the level of amplification of low-frequency voiced speech signals. This characteristic is one of the key features of the design which stated that the regenerated audio match the presbyacusis patient-specific pattern of hearing loss.

Since presbyacusis is a type of hearing disorder which is associated with aging process, this prototype has been tested on 10 senior citizens who suffer from hearing problem. Table 1 shows the feedbacks from the senior citizen after they have tried using the Android-based digital hearing aid prototype. Real-time test results show that $80 \%$ of the senior citizens are satisfied with the quality of the regenerated audio and $20 \%$ feels that there is no difference after using the prototype.

\section{CONCLUSION}

A digital hearing aid signal processing algorithm has been proposed in this paper. It is designed based on the specific hearing loss of the hearing disorder patient using inverse Fourier transform; besides, noise reduction feature is included in the digital algorithm design. Similar frequency response can be observed in the initial theoretical design and the MATLAB simulation results. The only difference between the design and simulation results is the amplification levels. The proposed algorithm provides flexibility to the users by allowing them to choose the desired amplification level. The proposed algorithm 
has been implemented in the Android-based smartphone and tested in real time. Results show that no obvious hearing latency can be detected. The proposed digital hearing aid is able to reduce noise level from the surrounding and the output processed speech match the patientspecific hearing loss.

\section{REFERENCES}

1. Costa MH. A complementary low-cost method for broadband noise reduction in hearing aids for medium to high SNR levels. Comput Biol Med 2014;46:29-41.

2. Global Burden of Disease Study 2013 Collaborators. Global, regional, and national incidence, prevalence, and years lived with disability for 301 acute and chronic diseases and injuries in 188 countries, 19902013: A systematic analysis for the global burden of disease study 2013. Lancet 2015;386:743-800.

3. Wrood SA, Baiee GK, Arif IS. Environmental exposures to low level of cadmium and lead and hearing impairment in Iraqi patients. Asian J Pharm Clin Res 2018;11:512-5.

4. Nelson JA. Coupling FM systems to high-technology digital hearing aids. J Educ Audiol 2002;9:13-20.

5. Dillier N, Frölich T, Kompis M, Bögli H, Lai WK. Digital signal processing (DSP) applications for multiband loudness correction digital hearing aids and cochlear implants. J Rehabil Res Dev 1993;30:95-109.

6. Ghamry NM. An FPGA implementation of hearing aids based on wavelet-packets. J Comput 2012;7:680-4

7. Kiefer J, von Ilberg C, Rupprecht V, Hubner-Egner J, Knecht R. Optimized speech understanding with the continuous interleaved sampling speech coding strategy in patients with cochlear implants: Effect of variations in stimulation rate and number of channels. Ann Otol Rhinol Laryngol 2000;109:1009-20.
8. Nogueira W, Büchner A, Lenarz T, Edler B. A psychoacoustic NofMtype speech coding strategy for cochlear implants. EURASIP J Appl Signal Process 2005;2005:3044-59.

9. Skinner MW, Clark GM, Whitford LA, Seligman PM, Staller SJ, Shipp DB, et al. Evaluation of a new spectral peak coding strategy for the nucleus 22 channel cochlear implant system. Am J Otol 1994;15 Suppl 2:15-27.

10. Panda G, Puhan NB. An improved block adaptive system for effective feedback cancellation in hearing aids. Digit Signal Process 2016;48:216-25

11. Ayllón D, Gil-Pita R, Rosa-Zurera M. Design of microphone arrays for hearing aids optimized to unknown subjects. Signal Process 2013;93:3239-50.

12. Kalathil S, Elias E. Efficient design of non-uniform cosine modulated filter banks for digital hearing aids. AEU Int J Electron Commun 2015;69:1314-20.

13. Chen C, Fan J, Hu X, Hei Y. A low power, high performance analog front-end circuit for $1 \mathrm{~V}$ digital hearing aid SoC. Circuits Syst Signal Process 2015;34:1391-404.

14. Stetzler T, Magotra N, Gelabert P, Kasthuri P, Bangalore S. Low Power Real-time Programmable DSP Development Platform for Digital Hearing Aids. Vol. 4. Phoenix: IEEE International Conference on Acoustics, Speech, and Signal Processing; 1999. p. 2339-42.

15. Ozdalga E, Ozdalga A, Ahuja N. The smartphone in medicine: A review of current and potential use among physicians and students. J Med Internet Res 2012;14:e128.

16. Steven SW. The Scientist and Engineer's Guide to Digital Signal Processing. California: California Technical Publisher; 1997.

17. Goh YH, Raveendran P, Jamuar SS. Robust speech recognition using harmonic features. IET Signal Process 2014;8:167-75

18. Available from: https:/www.chimehealth.co.uk/web/data/audiogramhearing-loss-examples-2.pdf. [Last accessed on $2014 \mathrm{Jul} 25$ ]. 